\title{
Treating Haglund's Deformity with percutaneous Achilles tendon decompression: a case series
}

\author{
Fabrizio Sergio ${ }^{1}$ \\ Ottorino Catani ${ }^{2}$ \\ Federico Fusini ${ }^{3}$ \\ Francesco Langella 4 \\ Giovanni Cautiero 5 \\ Ida Ponzo 5 \\ Fabio Zanchini ${ }^{5}$
}

1 Department of Orthopaedic and Traumatology, Casa di Cura S. Maria della Salute, Santa Maria Capuavetere, Caserta, Italy

2 Department of Foot Surgery, Casa di Cura Santa Rita, Avellino, Italy

3 Department of Orthopaedic and Traumatology, Orthopaedic and Trauma Center, University of Turin, Turin, Italy

4 IRCCS Istituto Ortopedico Galeazzi, Milan, Italy

5 Clinical Orthopaedics, University of Campania "Luigi Vanvitelli", Naples, Italy

\section{Corresponding author:}

Federico Fusini

Department of Orthopaedic and Traumatology

Orthopaedic and Trauma Center

University of Turin

Via G. Zuretti 29

10126 Turin, Italy

E-mail: fusinif@hotmail.com

\section{Summary}

Introduction: Haglund's syndrome is a deformity of the postero-superior and lateral heel-shaped profile commonly associated with inflammation of the retro-calcaneal bursa and characterized by pain and swelling. Aim of the study was to evaluate the reliability and effectiveness of minimally invasive percutaneous surgical procedure for Achilles tendon decompression.

Methods: From January 2014 to April 2016, a total of 21 continuous patients with Haglund's deformity (15 men, 6 women, mean age of 40 years) were enrolled. The regularization of calcaneous profile and borsectomy were performed in all cases. The results were evaluated both clinically with American Orthopaedic Foot and Ankle Society hindfoot score (AOFAS-hindfoot), Visual Analog Scale (VAS) before surgery and at 6 months' follow-up. Radiographic results were also collected using
Fowler-Philip before the surgery and one-month follow-up.

Results: The average AOFAS-hindfoot score increased from 50.57 to 97.42 ( $p<.00001)$. VAS score decreased from 6.85 to $0.19(p<.00001)$ and the mean Fowler-Philip angle decreased from $79,57^{\circ}$ to $53.76^{\circ}(p<.00001)$. In our series, no complications were observed and all patients were satisfied at last follow-up.

Conclusions: Our results suggest that Achilles decompression by percutaneous approach for painful Haglund's deformity is a reliable and effective surgical procedure.

Level of evidence: IV.

KEY WORDS: Achilles tendon decompression, Haglund's deformity, minimally invasive, percutaneous surgery.

\section{Introduction}

In 1928 Haglund described a condition of heel deformity, called "pump bump", clinically characterized by deformity and painful swelling at the level of posterosuperior and lateral portion of the calcaneus, where Achilles tendon takes its bony insertion ${ }^{1,2}$. The position of osseous deformity is involved in inflammation of retrocalcanear tendon bursa and subsequent Achilles tendinopathy ${ }^{3-6}$. This condition commonly affects women and athletes, in particular runners ${ }^{7}$.

Haglund's deformity may be a difficult and persistent problem and conservative treatments is often inadequate. The surgical approach is commonly indicated when conservative cares fail ${ }^{8,9}$.

A lot of surgical techniques were reported such as open, percutaneous or endoscopic approach. These surgical procedures are usually aimed to remove dorsolateral part of the calcaneus up to the insertion of the Achilles tendon and the inflamed retrocalcaneal bursa ${ }^{10}$. The right amount of bone decompression is correlated with clinical outcome. On one side, an inadequate or incomplete osteotomy often requires revision surgery, on the other side an excessive osteotomy may be cause of gastrocnemius and soleus muscular complex weakening ${ }^{9}$.

In our research, we adopted a percutaneous surgical approach to treat painful Haglund's deformity. Using a minimal skin incision, percutaneous techniques grant less soft tissue damage and less risk of wound and septic complications ${ }^{11,12}$. 
Our retrospective series aimed to demonstrate the safety and effectiveness of the percutaneous surgical technique in patients whit Haglund's deformity. We furthermore evaluated the clinical and radiographic outcome, along with complication rate.

\section{Materials and methods}

We present a series of 21 consecutive patients treated from January 2014 to April 2016 (15 males, 6 females) with mean age 40 years (range, 30-55). The demographic characteristics are listed in Table I.

All patients were affected with bilateral postero-superior/lateral calcaneus corner enlargement but monolateral symptomatic Haglund's deformity. They referred walking pain and difficulty when wearing shoes.

At the clinical examination, all patients presented a monolateral painful bony enlargement with calcaneal bursa inflammation. Severe acute pain followed pushing heel bump in all cases. American Orthopaedic Foot and Ankle Society - hindfoot scale (AOFAShindfoot) was used to evaluate surgical outcomes while Visual Analogue Scale (VAS) was used to in- vestigate the degrees of pain before and after surgical treatment.

Weight bearing X-ray was used to evaluate the bony exostosis at the postero-superior corner of the calcaneus (Fig. 1). In particular, Fowler-Philip angle on lateral view ( $F P a$ angle between the line drawn tangent to the postero-superior border of the calcaneus and the calcaneal tuberosity and the line drawn tangent to the inferior border of the calcaneus) was used to assess the amount of surgical correction for its capability to reflect the relation between the inferior and the posterior calcaneus.

FPa usually measures between 44 and $69^{\circ}$ while a degree greater than $75^{\circ}$ was systematically associated with Haglund's deformity ${ }^{13}$.

Before starting the surgical procedure, all patients were informed about the operative procedure, risks and potential complication and provided written informed consent and agreed to surgical procedure. AOFAS-hindfoot score (3 subsections: pain, 40 points; function, 50 points; alignment, 10 points) was evaluated before surgery and at last follow-up, X-ray was evaluated pre-operatively and one month after

Table I. Main demographic characteristics. Data in parentheses are percentages.

\begin{tabular}{ll}
\hline Participants $(\mathrm{N})$ & $21(100)$ \\
Female gender $(\mathrm{N})$ & $6(28,6)$ \\
Age (year) & 40 \\
$\quad$ Mean & 30 to 55 \\
$\quad$ Range & \\
Laterality & $10(47,6)$ \\
$\quad$ Left (main symptomatic) & $11(52,4)$ \\
$\quad$ Right (main symptomatic) &
\end{tabular}

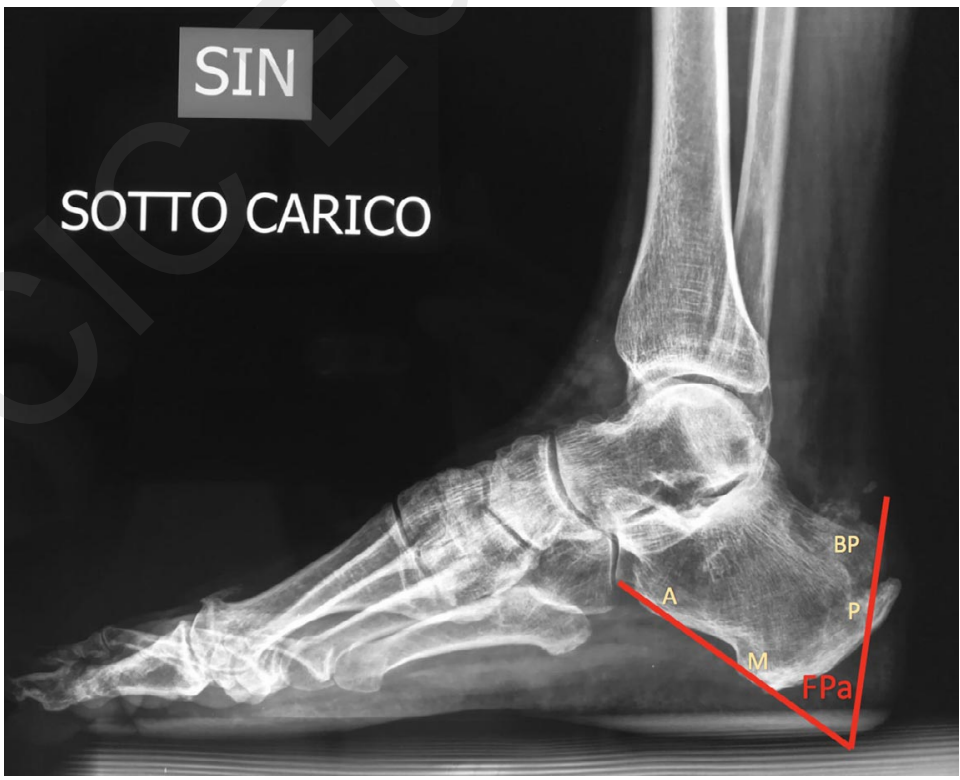

Figure 1. Pre-operative X-ray of a symptomatic patient, Fowler-Philip angle ( $\mathrm{FPa}$ ) measurement in a case of Haglund's deformity with concomitant presence of Achilles tendon calcification [FPa is the angle formed by the intersection of tangent line to the anterior tubercle $(\mathrm{A})$ and medial tuberosity $(\mathrm{M})$ with the line tangent to posterior surface of the bursal projection (BP) and the posterior tuberosity $(P)]$. 
surgery. Return to daily life activity, in weeks, was also recorded.

Mean follow-up was $16,76 \pm 5,25$ (Mean \pm SD) months. The article meets the ethical standards of the journal ${ }^{14}$. Informed consent was obtained by all participants.

\section{Surgical procedure}

The patient is positioned on the operating table in lateral decubitus under spinal anaesthesia. An incision of $5 \mathrm{~mm}$ is made on the lateral side of the heel, just ahead the insertion of Achilles tendon, over the dorsal surface of heel. The incision is continued down to exostosis base. A small rasp is used to remove the fibrous tissue and periosteum from the surface. A cylindrical burr 3.1-15 (Shannon 44, Miltex Instrument, Co., Inc., Rietheim-Weilheim, Germany) is inserted to remove the exostosis with a sweeping movement. Burr must be inserted without excessive angulation. Burr speed should be approximately 4000 to $5000 \mathrm{rpm}$ and physiological solution is used to irrigate surrounding tissues to avoid bone necrosis or other damages. During this phase, a second hand is positioned on the medial pretendinous loggia to check the position of the tip of the drill. This manoeuvre may help to reduce the risk of the vascular or nerve damage. Under X-ray fluoroscopic control (BJ-2J portable fluoroscopic device, Shanghai Bojin Electric Instruments \& Devices Co., Ltd), attention must be paid to save as much as possible the bone tissue of the enthesis zone and do not violate the anterior surface of the Achilles tendon. After exostosis removal, light pressure is ap- plied to the area to eject the bone particles. Elimination of bone is completed using a small rasp when it is not possible to extract all the bone growth. An intraoperative fluoroscopic check is made to verify the complete deformity removal (Fig. 2).

\section{Post-surgical care}

Full weight bearing is permitted immediately, assisted by crutches during the first 2 weeks after surgery. We recommended to use comfortable shoe without heel support for 2-4 weeks. Since immediate weight bearing, no physiotherapy was required. We remove the dressing after two weeks. Clinical and radiological control is performed after 1 month and 6 months from surgical procedure (Figs. 3, 4).

\section{Statistical analysis}

Data were reported as mean \pm SD and confidence interval (Cl). Mann-Whithney $U$ test was used to assess changes between pre and post-operative results in AOFAS-hindfoot score, VAS and FPa. Correlation between AOFAS-hindfoot score results and decreasing of $\mathrm{FPa}$ and between decreasing of FPa and VAS was assessed through Pearson correlation coefficient.

Level of significance was set at $p<0.05$.

\section{Assessors}

Two of us (F.F. and F.L.) performed radiographic measure, data collection, statistical analysis, manuscript preparation. Two others (G.C. and I.P.) performed data collection, radiographic measure and language editing. Two of us (O.C. and F.Z.) per-

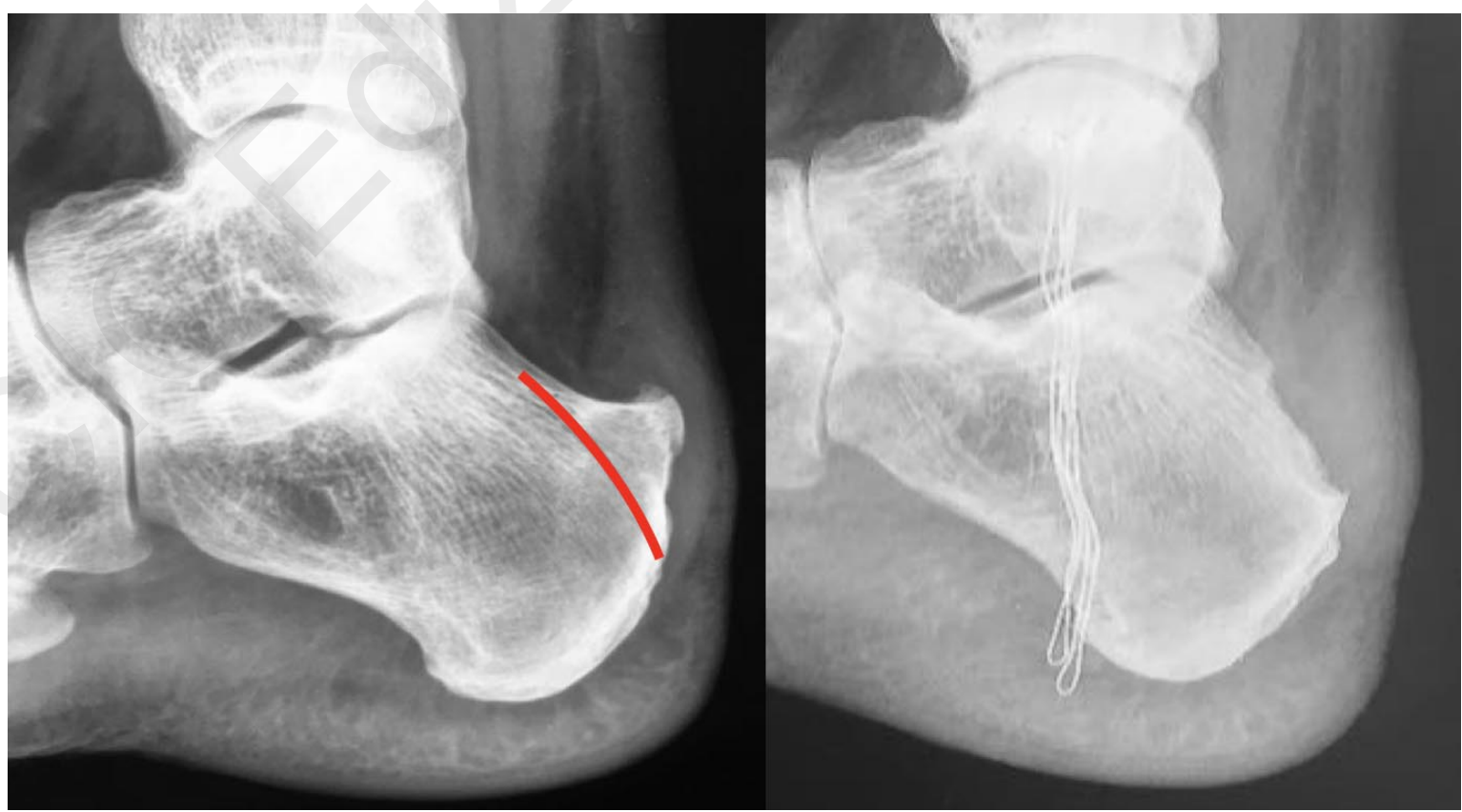

Figure 2. Pre-operative planning (left) and intra-operative (right) X-ray showing satisfactory deformity correction. 


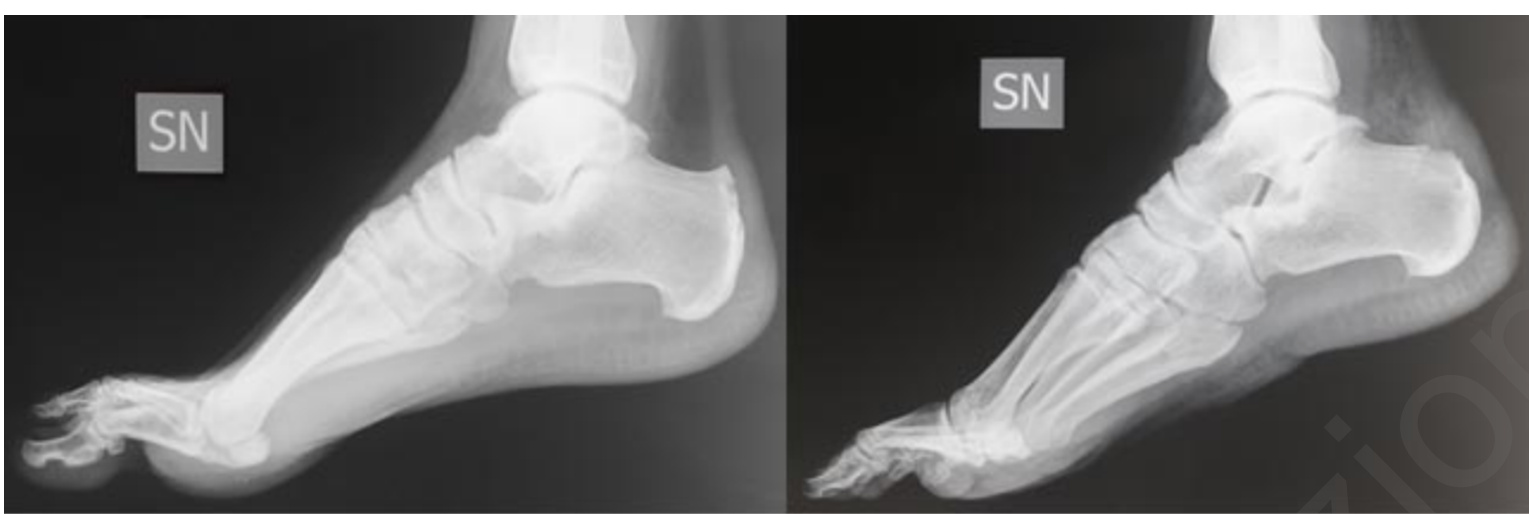

Figure 3. Pre-operative (left) and post-operative (right) X-ray at 6 months follow-up showing complete deformity correction.

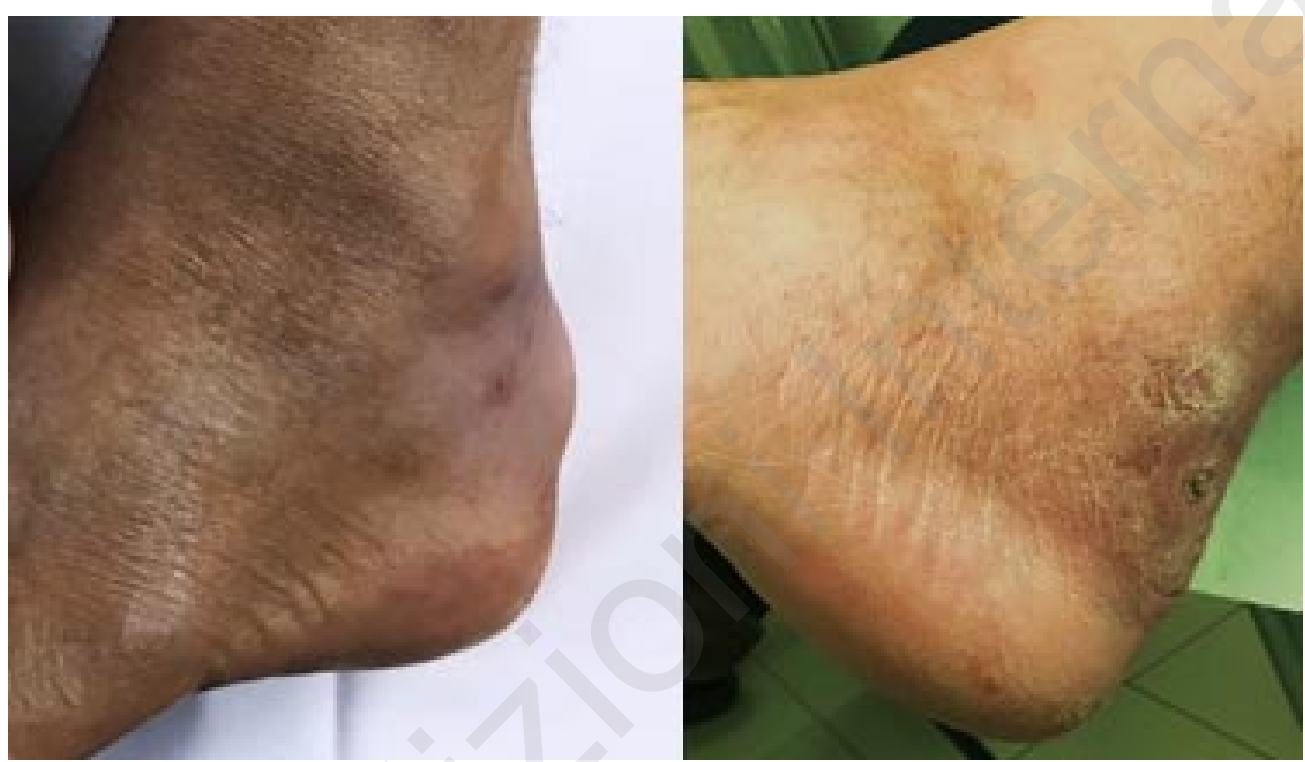

Figure 4. Clinical evaluation of "pump bump" at pre-operative (left) and 14 days (right) follow-up.

formed outcome assessment and study design. Finally, one of us (F.S.) performed radiographic measures and surgery.

\section{Results}

At last follow-up, mean AOFAS-hindfoot scale increased from $50.57 \pm 7.39(95 \% \mathrm{Cl} 47.37$ to 53.77$)$ pre-operatively to $97.43 \pm 4.30(95 \% \mathrm{Cl} 95.59$ to $99.27)$ with $p<.00001$. The major changes were reported in the pain subsection of AOFAS-hindfoot score, while no changes were found in ankle alignment.

One month after treatment mean FPa decreased from $79,57 \pm 2.46(95 \% \mathrm{Cl} 78.47$ to 80.67$)$ to $53.76 \pm 6.03$ $(95 \% \mathrm{Cl} 51.18$ to 56.34$)$ and $p<0.00001$. VAS value decreased from $6.85 \pm 0.91(95 \% \mathrm{Cl} 6.47$ to 7.25$)$ to $0.19 \pm 0.4(95 \% \mathrm{Cl} 0.02$ to 0.36$)$ and $p<0.00001$. Clinical and radiographic outcomes are resumed in Table II and III respectively.
The Pearson correlation coefficient between the increasing of AOFAS-hindfoot score and decreasing of FPa was 0.52 , while it reached the value of 0.73 between the decreasing of FPa and VAS.

We reported no major complications. Some patients referred only little discomfort in the first month after treatment at the level of cutaneous scar. This symptom reduced day by day practicing scar mobilization and anti-adherence massages without use of NSAID. Also, no recurrence was reported until last follow-up. Patients return to daily life activities in mean $4 \pm 0.77$ weeks (range 3-6 weeks).

\section{Discussion}

In Haglund's deformity, the goal of surgical treatment is to remove the bone bump that irritates Achilles tendon. An adequate bone resection during the calcaneoplasty procedure is essential to reduce painful symptoms ${ }^{15,16}$. The key points to achieve the best 
Table II. Pre and post-operative functional outcome of patients enrolled in the study. AOFAS, American Orthopaedic Foot and Ankle Society Hind-foot Score; VAS, Visual Analog Scale; Cl, confidence interval; SD, standard deviation.

\begin{tabular}{lll}
\hline & AOFAS & VAS \\
\hline $\begin{array}{cl}\text { Pre-operative } \\
\text { Mean } \pm \text { SD }\end{array}$ & $50.57 \pm 7.39$ & $6.85 \pm 0.91$ \\
$95 \% \mathrm{Cl}$ & 47.37 to 53.77 & 6.47 to 7.25 \\
Post-operative & & \\
Mean \pm SD & $97.43 \pm 4.30$ & $0.19 \pm 0.4$ \\
$95 \% \mathrm{Cl}$ & 95.59 to 99.27 & 0.02 to 0.36 \\
$p$-value & $<0.00001$ & $<.00001$ \\
\hline
\end{tabular}

Table III. Radiological assessment of pre and postoperative $\mathrm{FPa}$ of patients underwent percutaneous surgery.

\begin{tabular}{cl}
\hline & FPa $\left(^{\circ}\right)$ \\
\hline Pre-operative & \\
Mean \pm SD & $79.57 \pm 2.46$ \\
$95 \% \mathrm{Cl}$ & 78.47 to 80.67 \\
Post-operative & \\
Mean \pm SD & $53.76 \pm 6.03$ \\
$95 \% \mathrm{Cl}$ & 51.18 to 56.34 \\
$p$-value & $<0.00001$ \\
\hline
\end{tabular}

FPa, Fowler-Philip angle; $\mathrm{Cl}$, confidence interval; SD, standard deviation.

clinical outcome is the right surgical indication. This deformity can be approached by open or by minimally invasive procedures: arthroscopic or percutaneous. When is present a condition of degenerative pattern of the Achilles tendon, an open approach is considered the gold standard. Indeed, the open procedure allows performing a complete curettage or a reinforcement of the tendon when required ${ }^{17}$. Arthroscopic approach counts several advantages ${ }^{18}$ : joint cartilage and ligament evaluation, removal of loose intrarticular bodies, assessment of synovitis, low rate of complications, blood loss, perioperative pain and a faster recovery than open surgery.

As the open approach, the arthroscopic path through the posterior portals is not free from complications ${ }^{19-21}$ as synovitis, ganglia formations or portal cysts, injuries of the superficial peroneal tendon, Achilles tendon rupture, nor even medial vascular nerve structures and required a significant learning curve.

To the best of our knowledge, this is the first series considering the percutaneous correction of Haglund's deformity, with particular attention on clinical and radiographic outcomes and their correlation. The results of our series showed a significant correlation between post-operative AOFAS-hindfoot or VAS and the FPa correction. This angle varies with the amount of retro calcaneal bone spur resection ${ }^{22}$. Insufficient bone resection is related to the recurrence of symptoms and other surgical complications such as Achilles tendon rupture, ankle stiffness or scar formation with nerve entrapment ${ }^{23}$. On the other hand, an aggressive removal or damage of Achille's enthesis can hesitate in severe complication. The gold standard procedure for bone resection is open surgery that is capable to reach better surgical correction than endoscopic or minimally invasive technique. Despite this, results in literature after open resection and bursectomy are discordant ${ }^{24.25}$. Anderson et al. ${ }^{16}$ retrospectively reviewed 30 patients (31 feet) who underwent the tendon splitting approach and compared their results with 32 previous patients (35 feet) who had a lateral incision. Comparing our results with those of pre-operative AOFAS-hindfoot score were similar while our AOFAS-hindfoot post-operative results were considerably better of both groups. Also return to daily activity was substantially faster in our series when compared with Anderson's results. Our technique, using a tissue-sparing incision didn't affect Achilles tendon tissue and didn't require long ankle immobilization. In particular, during the surgical procedure, we reduce the exposition of the Achilles tendon and we systematically preserved the osseous portion of the enthesis to decrease the risk of tendon injury. The respect of the enthesis offers the possibility of an early weight-bearing maintaining a minimally plantar flexion for two weeks and reducing the risk of post-operative detachments of the Achilles tendon ${ }^{26,27}$. In a previous study, Brunner et al. ${ }^{28}$ adopted an open calcaneal osteotomy in unresponsive Haglund's deformity. In his series, he founded a worse AOFAS-hindfoot score at last follow-up and also a less satisfaction from treated patients. In fact, about $15 \%$ of patients did not recommend this procedure due to long immobilization (4 weeks before weight-bearing and 8 weeks before cast removal). In our series, the weight-bearing was immediate with tolerance and assisted with crunch for the first 4 weeks (range 3-6 weeks). This differences in post-op- 
erative strategy is mainly due to lack of procedure on Achilles enthesis.

According to Vernois et al. ${ }^{29}$, a meticulous approach during both shaving and cleaning must be done. During the reaming procedure, the surgeons must pay attention to not aggress too deeply the calcaneus bone, to prevent bone impairment with subsequent fracture or damage of Achilles tendon enthesis. Also a direct damage of Achilles tendon is possible, with a subsequent tendon rupture. For these reasons, a systematic intra-operative fluoroscopic control is essential. Bone paste must be continuously removed to avoid inflammatory response and recurrence. Moreover, despite percutaneous approach seems to be simple and quick with low complications, a long training curve is necessary to acquire the correct tactile sensibility during the reaming procedure. This last point is essential since a direct view is not possible.

Some limitation to our study must be reported: some are intrinsic to the study design, such as the lack of a control group or randomization and the retrospective design. Despite the excellent results of the clinical score at last follow-up, we reported also a not homogeneous follow-up time (mean follow-up 16.76 \pm 5.25 months, range 9-26 months). Giving that, we have no available data on clinical outcomes after such period, especially for those patients with shorter follow-up; and data on recurrence were not investigated. Furthermore, the low number of patients and the lack of a priori sample size calculation must be reported. Other limitations are related to the surgical procedure: the data were collected from a single experienced foot surgeon and the reliance on fluoroscopy for this procedure depends on the skills of the surgeon. The experience acquired, and the rapidity of this procedure makes it possible to verify the effectiveness of the treatment with very few X-ray images. -In particular, a portable X-ray fluoroscopy equipment in our series allows reducing the radiation exposure. Moreover, the lack of publishing data on percutaneous technique and the low number of open or less invasive approach didn't allow an extensive comparison with our study in terms of clinical and radiographic outcomes.

\section{Conclusions}

In conclusion, the percutaneous correction of Haglund's deformity is an effective and safe procedure whit excellent clinical outcome and good correlation with radiological measure. The lack of common complication of open surgery such as painful scar with nerve entrapment or surgical wound dehiscence in association with excellent patients' satisfaction and fast return to daily activities make the percutaneous approach a valuable treatment choice in the management of Haglund's deformity.

\section{Compliance with ethical standards}

\section{Conflict of interest}

The Authors declare that they have no conflict of interest.

\section{Patient consent}

All patients gave informed consent prior to inclusion in the study.

\section{Funding}

No benefits in any form have been received or will be received from a commercial party related directly or indirectly to the subject of this article.

\section{References}

1. Haglund P. Beitrag zur Klinik der Achillessehne. Zeitschr Orthop Chir. 1928:49:49-58.

2. Maffulli N, Denaro V, Loppini M. Haglund's deformity. Foot ankle Int [Internet]. 2012 Sep [cited 2018 May 14];33(9):807-808. Author reply 808 . Available from: http://journals.sagepub. com/doi/10.3113/FAl.2012.0807

3. Pavlov H, Heneghan MA, Hersh A, Goldman AB, Vigorita V. The Haglund syndrome: initial and differential diagnosis. Radiology. 1982;144(1):83-88.

4. Busilacchi A, Olivieri M, Ulisse S, Gesuita R, Skrami E, Lording $\mathrm{T}$, et al. Real-time sonoelastography as novel follow-up method in Achilles tendon surgery. Knee Surg Sports Traumatol Arthrosc. 2016;24(7)

5. Fusini F, Langella F, Busilacchi A, Tudisco C, Gigante A, Massé $A$, et al. Real-time sonoelastography: principles and clinical applications in tendon disorders. A systematic review. MLTJ. 2017;7(3):467-477.

6. Fusini F, Bisicchia S, Bottegoni C, Gigante A, Zanchini F, Busilacchi A. Nutraceutical supplement in the management of tendinopathies: A systematic review. MLTJ. 2016;6(1).

7. Jerosch J, Schunck J, Sokkar SH. Endoscopic calcaneoplasty (ECP) as a surgical treatment of Haglund's syndrome. Knee Surg Sports Traumatol Arthrosc. 2007;15(7):927-934.

8. Heneghan MA, Pavlov H. The Haglund Painful Heel Syndrome Experimental Investigation of Cause and Therapeutic Implications. Clin Orthop Relat Res. 1984;187:228-234.

9. Sella EJ, Caminear DS, McLarney EA. Haglund's syndrome. J Foot Ankle Surg. 1998;37(2):110-114.

10. Roth KE, Mueller R, Schwand E, Maier GS, Schmidtmann I, Sariyar M, et al. Open versus endoscopic bone resection of the dorsolateral calcaneal edge: a cadaveric analysis comparing three dimensional CT scans. J Foot Ankle Res. 2015/01/23. 2014;7(1):56.

11. Fusini $F$, Langella $F$, Catani $O$, Sergio F, Zanchini F. Mini-Invasive Treatment for Brachymetatarsia of the Fourth Ray in Females: Percutaneous Osteotomy With Mini-Burr and External Fixation-A Case Series. J Foot Ankle Surg. 2017.

12. Oliva F, Piccirilli E, Tarantino U, Maffulli N. Percutaneous release of the plantar fascia. New surgical procedure. MLTJ. 2017;7(2):338.

13. Fowler A, Philip JF. Abnormality of the calcaneus as a cause of painful heel its diagnosis and operative treatment. $\mathrm{Br} \mathrm{J}$ Surg 1945;32(128):494-498.

14. Padulo J, Oliva F, Frizziero A, Maffulli N. Muscles, Ligaments and Tendons Journal - Basic principles and recommendations in clinical and field science research: 2016 update. MLTJ. 2016;6(1):1-5. 
15. Roth KE, Mueller R, Schwand E, Maier GS, Schmidtmann I, Sariyar M, et al. Open versus endoscopic bone resection of the dorsolateral calcaneal edge: a cadaveric analysis comparing three dimensional CT scans. J Foot Ankle Res. 2014;7(1):56.

16. Anderson JA, Suero E, O'Loughlin PF, Kennedy JG. Surgery for retrocalcaneal bursitis: a tendon-splitting versus a lateral approach. Clin Orthop Relat Res. 2008/05/10. 2008;466(7): 1678-1682.

17. Maffulli N, Del Buono A, Testa V, Capasso G, Oliva F, Denaro V. Safety and outcome of surgical debridement of insertional Achilles tendinopathy using a transverse (Cincinnati) incision. J Bone Joint Surg Br 2011 Nov 1;93(11):1503-1507.

18. Parvizi J, Kim GK. Ankle Arthroscopy. In: High Yield Orthopaedics. Elsevier. 2010;25.

19. Deng DF, Hamilton GA, Lee M, Rush S, Ford LA, Patel S. Complications Associated with Foot and Ankle Arthroscopy. J Foot Ankle Surg. 2012 May;51(3):281-284.

20. Nickisch F, Barg A, Saltzman CL, Beals TC, Bonasia DE, Phisitkul $\mathrm{P}$, et al. Postoperative Complications of Posterior Ankle and Hindfoot Arthroscopy. J Bone Joint Surg Am. 2012 Mar 7;94(5):439-446.

21. Gasparetto F, Collo G, Pisanu G, Villella D, Drocco L, Cerlon $\mathrm{R}$, et al. Posterior ankle and subtalar arthroscopy: indications, technique, and results. Curr Rev Musculoskelet Med. 2012 Jun 17;5(2):164-170.
22. Bulstra GH, van Rheenen TA, Scholtes VA. Can We Measure the Heel Bump? Radiographic Evaluation of Haglund's Deformity. J Foot Ankle Surg. 2015;54(3):338-340.

23. Kucuksen S, Karahan AY, Erol K. Haglund syndrome with pump bump. Med Arch. 2013/02/16. 2012;66(6):425-427.

24. Vaishya R, Agarwal AK, Azizi AT, Vijay V. Haglund's Syndrome: A Commonly Seen Mysterious Condition. Cureus. 2016;8(10):e820.

25. Jerosch J. Endoscopic calcaneoplasty. Foot Ankle Clin. 2015;20(1):149-165.

26. Heller W. [Achilles tendon rupture following surgery for a Haglund heel]. Z Orthop Ihre Grenzgeb. 1971 Jul;109(3):534537.

27. Lai Wei Hong S, Tang Qian Ying C, Thwin L, Thevendran G. Return to Sport and Physical Activity After Calcaneoplasty for Insertional Achilles Tendinosis. J Foot Ankle Surg. 2016 Nov;55(6):1190-1194.

28. Brunner J, Anderson J, O'Malley M, Bohne W, Deland J, Kennedy J. Physician and patient based outcomes following surgical resection of Haglund's deformity. Acta Orthop Belg. 2006/02/08. 2005;71(6):718-723.

29. Vernois J, Redfern D, Ferraz L, Laborde J. Minimally Invasive Surgery Osteotomy of the Hindfoot. Clin Pod Med Surg. 2015/06/29. 2015;32(3):419-434. 